\title{
Natural Convection Flow of MHD Micropolar Fluid Along a Vertical Wavy Surface
}

\author{
S. Sultana and Nepal C. Roy* \\ Department of Mathematics, Dhaka University, Dhaka-1000, Bangladesh
}

(Received: 18 July 2016; Accepted: 2 March 2017)

\begin{abstract}
We study the boundary layer characteristics of natural convection flow of an electrically conducting micropolar fluid along a vertical wavy surface. The dimensionless governing equations have been solved numerically. Results are presented in terms of the local skin-friction coefficient, the local Nusselt number and the local couple stress with the variation of amplitude-wavelength ratio, magnetic parameter, vortex viscosity parameter and spin-gradient viscosity parameter. Due to increase of the amplitude wave-length ratio, the skin-friction and the couple stress are found to decrease whereas the Nusselt number increases. The skin friction and the couple stress increase but the Nusselt number decreases for increasing values of vortex viscosity parameter. In addition, when the spin-gradient viscosity is increased, the maximum values of the Nusselt number and the couple stress significantly increase but the skin-friction decreases. The magnetic parameter considerably reduces the skin-friction, Nusselt number and couple stress.
\end{abstract}

\section{Introduction}

The fluids with microstructures are called micropolar fluids. These belong to a class of fluids with non-symmetric stress tensor. Physically, this fluid consists of rigid, randomly oriented particles with their own spins and micro rotations, suspended in a viscous medium. Micropolar fluids have attracted much attention of many researches due to their industrial and engineering applications. Examples of micropolar fluids are ferrofluids, polymeric fluids, liquid crystals, animal blood, dirty oils, exotic lubricants, colloidal suspensions and so on.

The Navier-stokes equations of Newtonian and nonNewtonian theory cannot explain the characteristics of several physiological fluids which exhibit microscopic effects arising from the local structures and micromotions of the fluid elements. For this reason, many constitutive models have been suggested by several researchers. Among these models the theory of micropolar fluids and thermomicropolar fluids developed by Eringen ${ }^{1,2}$ has attracted considerable attentions. Ariman et al. ${ }^{3,4}$ provided an excellent review about micropolar fluid mechanics. However, $\mathrm{Yao}^{5}$ used the transformation method to study the natural convection flow along a sinusoidal wavy surface. Chiu and $\mathrm{Chou}^{6}$ investigated the free convection in the boundary layer flow of a micropolar fluid along a vertical wavy surface. A simple transposition theorem and cubic spline collocation numerical method have been used to solve the governing equations. They observed that increasing the micropolar fluid parameter results in decreasing heat transfer rates and increasing local skin friction as well as hydrodynamic and thermal boundary layer thicknesses. Also, transient analysis of natural convection along a vertical wavy surface in micropolar fluids has been studied by Chiu and $\mathrm{Chou}^{7}$. Moreover, Ishak et al. ${ }^{8}$ studied MHD stagnation-point flow of a micropolar fluid with prescribed wall heat flux. A steady two-dimensional MHD mixed convection flow of micropolar fluid toward a stretching/shrinking vertical surface with prescribed surface heat flux has been examined by Adhikari ${ }^{9}$.

The objective of the present work is to analyze the natural convection flow along a vertical wavy surface in the presence of magnetic field. The governing equations are first transformed into a non-dimensional form by using appropriate non-dimensional variables. These equations are then transformed into a system of non linear partial differential equations and finally the equations are solved numerically by using finite difference method. Numerical results of local skin-friction coefficient, local Nusselt number and local couple stress under the effect of amplitude-wavelength ratio, magnetic parameter, vortex viscosity parameter and spin-gradient viscosity parameter are presented.

\section{Mathematical Formulation}

We consider a vertical wavy surface immersed in a micropolar fluid at the ambient temperature $T_{\infty}$. The schematic diagram of the flow configuration is shown in Fig. 1. It is assumed that the surface temperature of the wavy plate, $T_{w}$, is greater than the ambient temperature, $T_{\infty}$. We also consider that the surface is described by $y=\bar{\sigma}(x)=a \sin (2 \pi x / L)$ where $a$ is the dimensional amplitude of wavy surface, $L$ the characteristic length scale associated with the waves and the origin of the coordinate system is placed at the leading edge of the vertical surface.

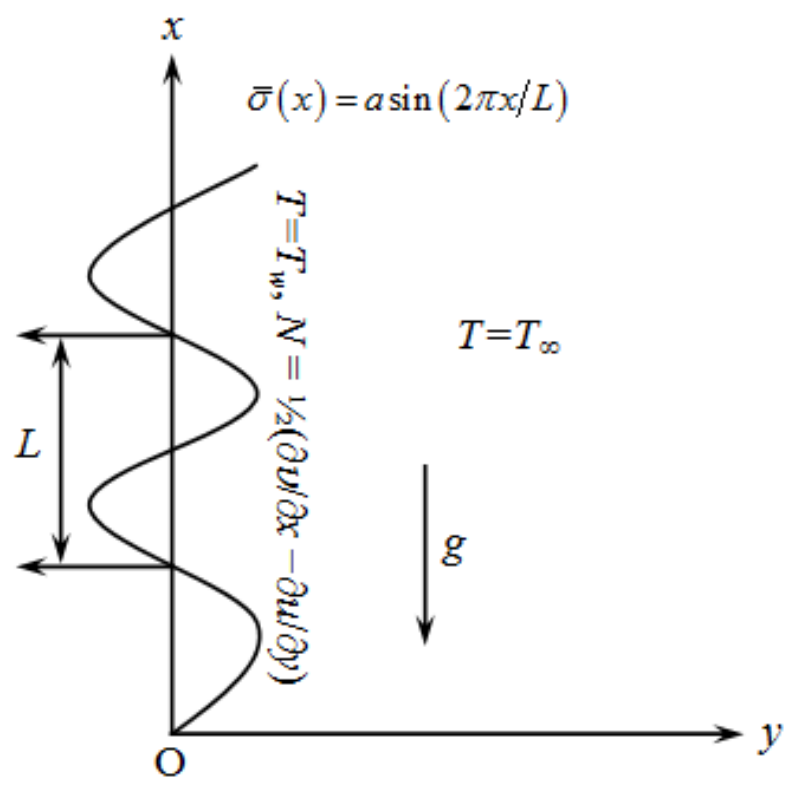

Fig. 1. Schematic diagram of the flow

Let us consider a two-dimensional, steady, laminar, incompressible micropolar fluid flow along a vertical wavy surface. Following ${ }^{6}$, the dimensionless governing equations for such a flow in the presence of magnetic field can be expressed as: 


$$
\frac{\partial u^{*}}{\partial x^{*}}+\frac{\partial v^{*}}{\partial y^{*}}=0
$$

$u^{*} \frac{\partial u^{*}}{\partial x^{*}}+v^{*} \frac{\partial u^{*}}{\partial y^{*}}=-\frac{\partial p^{*}}{\partial x^{*}}+\sigma^{\prime} \frac{\partial p^{*}}{\partial y^{*}} G r^{1 / 4}$

$+\left(1+\sigma^{\prime 2}\right)(1+K) \frac{\partial^{2} u^{*}}{\partial y^{* 2}}+\theta^{*}+K \frac{\partial h^{*}}{\partial y^{*}}-M u^{*}$

$u^{* 2} \sigma^{\prime \prime}+\sigma^{\prime}\left(u^{*} \frac{\partial v^{*}}{\partial x^{*}}+v^{*} \frac{\partial v^{*}}{\partial y^{*}}\right)=-G r^{\frac{1}{4}} \frac{\partial p^{*}}{\partial y^{*}}$

$+\sigma^{\prime}\left(1+\sigma^{\prime 2}\right)(1+K) \frac{\partial^{2} u^{*}}{\partial y^{* 2}}+K \sigma^{\prime} \frac{\partial h^{*}}{\partial y^{*}}$

$u * \frac{\partial h^{*}}{\partial x^{*}}+v^{*} \frac{\partial h^{*}}{\partial y^{*}}=\lambda\left(1+\sigma^{2}\right) \frac{\partial^{2} h^{*}}{\partial y^{* 2}}$

$-K B\left\{\left(1+\sigma^{\prime 2}\right) \frac{\partial u^{*}}{\partial y^{*}}+2 h^{*}\right\}$

$u \frac{\partial \theta^{*}}{\partial x^{*}}+v^{*} \frac{\partial \theta^{*}}{\partial y^{*}}=\frac{1}{\operatorname{Pr}}\left(1+\sigma^{\prime 2}\right) \frac{\partial^{2} \theta^{*}}{\partial y^{* 2}}$

where the dimensionless variables are defined as

$$
\begin{aligned}
& x^{*}=\frac{x}{L}, y^{*}=\frac{y-\bar{\sigma}}{L} G r^{1 / 4}, \\
& u^{*}=\frac{\rho L}{\mu G r^{1 / 2}} u, v^{*}=\frac{\rho L}{\mu G r^{1 / 4}\left(v-\sigma^{\prime} u\right),} \\
& p^{*}=\frac{\rho L^{2}}{\mu^{2} G r} p, \quad \sigma=\frac{\bar{\sigma}}{L}, N=\frac{v G r^{3 / 4}}{L^{2}} h^{*}, \\
& \theta=\frac{T-T_{\infty}}{T_{w}-T_{\infty}}, \quad G r=\frac{g \beta_{T}\left(T_{w}-T_{\infty}\right) \rho^{2} L^{3}}{\mu^{2}},
\end{aligned}
$$

and $\operatorname{Pr}=v / \alpha$ is the Prandtl number, $G r=g \beta_{T}\left(T_{w}-T_{\infty}\right) \rho^{2} L^{3} / \mu^{2}$ is the Grashof number, $M=\sigma_{c} B_{0}{ }^{2} L G r^{-1 / 2} / \rho^{2}$ is the dimensionless magnetic parameter, $K=\kappa / \mu$ is the vortex viscosity parameter, $B=L^{2} /\left(j G r^{1 / 2}\right) \quad$ is the material parameter and $\lambda=\gamma /(\mu j)$ is the spin-gradient viscosity parameter.

In the above variables and parameters, $u$ and $v$ are the velocity components in the $x$ and $y$ directions respectively, $T$ is the temperature of the fluid, $p$ is the pressure, $\sigma_{c}$ is the electrical conductivity, $D$ is the diffusivity, $\rho$ is the density of the fluid, $N$ is the component of the micro-rotation vector normal to the $x y$-plane, $\gamma$ is the spin gradient viscosity, $\kappa$ is the thermal conductivity and $g$ is the acceleration due to gravity.

It should be mentioned that equation (11) indicates that $\partial p^{*} / \partial y^{*}$ is of order $G r^{1 / 4}$, which implies that the lower order pressure gradient along the $x$ axis is determined from the inviscid solution. However, for the present problem this gives $\partial p^{*} / \partial x^{*}=0$. Further, we multiply equation (11) by $\sigma^{\prime}$ and the resulting equation is added to equation (10) in order to eliminate the term $G r^{1 / 4}\left(\partial p^{*} / \partial y^{*}\right)$ from equations (10) and (11). After some manipulation, we get

$$
\begin{aligned}
& u^{*} \frac{\partial u^{*}}{\partial x^{*}}+v^{*} \frac{\partial u^{*}}{\partial y^{*}}+\frac{\sigma^{\prime} \sigma^{\prime \prime} u^{* 2}}{1+\sigma^{\prime 2}} \\
& =\left(1+\sigma^{\prime 2}\right)(1+K) \frac{\partial^{2} u^{*}}{\partial y^{* 2}}+\frac{\theta^{*}}{1+\sigma^{\prime 2}} \\
& +K \frac{\partial h^{*}}{\partial y^{*}}-\frac{M u^{*}}{1+\sigma^{\prime 2}}
\end{aligned}
$$

The boundary conditions become

at $y=0, u^{*}=v^{*}=0$ and $h^{*}=-\frac{1}{2}\left(1+\sigma^{2}\right) \frac{\partial u^{*}}{\partial y}$

at $y=\infty, u^{*}=0, \theta=0$ and $h^{*}=0$

We use the following transformation to remove the singularity

$$
\begin{aligned}
& X=x^{*}, Y=\left(4 x^{*}\right)^{-1 / 4} y^{*}, \\
& \psi=\left(4 x^{*}\right)^{3 / 4} f(X, Y, \zeta), h^{*}=\left(4 x^{*}\right)^{1 / 4} H
\end{aligned}
$$

where $\psi$ is the stream function which is defined by $(u, v)=(\partial \psi / \partial y,-\partial \psi / \partial x)$. The above mentioned equation then become

$$
\begin{aligned}
& 4 X f^{\prime} \frac{\partial f^{\prime}}{\partial X}-\left(3 f+4 X \frac{\partial f}{\partial X}\right) f^{\prime \prime} \\
& +\frac{1}{\left(1+\sigma^{\prime 2}\right)} M f^{\prime}+\left(2+\frac{4 X \sigma^{\prime} \sigma^{\prime \prime}}{1+\sigma^{\prime 2}}\right) f^{\prime 2} \\
& =\left(1+\sigma^{\prime 2}\right)(1+K) f^{\prime \prime \prime}+\frac{\theta}{\left(1+\sigma^{\prime 2}\right)}+K H^{\prime}
\end{aligned}
$$




$$
\begin{aligned}
& 4 X f^{\prime} \frac{\partial H}{\partial X}-\left(3 f+4 X \frac{\partial f}{\partial X}\right) H^{\prime} \\
& +\left\{f^{\prime}+2 K B(4 X)^{1 / 2}\right\} H=\lambda\left(1+\sigma^{2}\right) H^{\prime \prime} \\
& -K B\left(1+\sigma^{\prime 2}\right)(4 X)^{1 / 2} f^{\prime \prime} \\
& 4 X f^{\prime} \frac{\partial \theta}{\partial X}-\left(3 f+4 X \frac{\partial f}{\partial X}\right) \theta^{\prime}=\frac{1+\sigma^{\prime 2}}{\operatorname{Pr}} \theta^{\prime \prime}
\end{aligned}
$$

subject to the boundary conditions

$$
f=f^{\prime}=0, \theta=1 \text { and } H=-\frac{1}{2}\left(1+\sigma^{2}\right) f^{\prime \prime} \quad \text { at } Y=0
$$

$f^{\prime}=0, \theta=0$ and $H=0$ as $Y \rightarrow \infty$

Here primes denote differentiation with respect to $Y$. The equations (11)-(15) have been solved employing finite difference method ${ }^{10}$.

The local skin-friction, the local Nusselt number and the local couple stress are of great physical importance and these are defined respectively as

$$
\begin{aligned}
& C_{f}=\frac{2 \mu}{\rho \tilde{U}^{2}}\left[\frac{\partial u}{\partial y}+\frac{\partial v}{\partial x}+x\left(\frac{\partial u}{\partial y}+N\right)\right]_{y=0}, \\
& N u=-\frac{L}{k} \frac{k}{T_{w}-T_{\infty}}\left(\frac{\partial T}{\partial n}\right)_{y=0}, \\
& m_{N}=\frac{L \gamma}{j \rho \tilde{U}^{2}}\left(\frac{\partial N}{\partial n}\right)_{y=0}
\end{aligned}
$$

where $\tilde{U}=\mu G r^{1 / 2} /(\rho L)$ is a characteristic velocity and the shearing stress on the wavy surface is

Substituting the relations (6) and (10) into equation (16), we have

$$
\begin{aligned}
& C_{f x}=(2+K)\left(1-\sigma^{\prime 2}\right)\left(\frac{\partial U}{\partial Y}\right)_{Y=0}, \\
& N u_{x}=-\left(1+\sigma^{\prime 2}\right)^{1 / 2}\left(\frac{\partial \theta}{\partial Y}\right)_{Y=0}, \\
& m_{N}=\lambda\left(\frac{\partial H}{\partial Y}\right)_{Y=0}
\end{aligned}
$$

where

$$
C_{f x}=\left(\frac{4 X}{G r}\right)^{-1 / 4} C_{f}, N u_{x}=\left(\frac{G r}{4 X}\right)^{-1 / 4} N u
$$

\section{Results and Discussion}

The present problem that accounts for the effect of magnetic field on the local skin-friction, local Nusselt number and local couple stress of an incompressible micropolar fluid over a vertical wavy surface has been solved numerically. Numerical calculations are accomplished for the vertical wavy surface prescribed by $\bar{\sigma}(x)=a \cdot \sin (2 \pi x / L)$ or in dimensionless form $\sigma(X)=\alpha \cdot \sin (2 \pi X)$ where $\alpha=a / L$ is the amplitude-wavelength ratio. The values of the parameters are taken as $\operatorname{Pr}=0.1, \lambda=13.5, \alpha=0.05, M=0.5$, $K=5.0$ except the variation of one of them.

The effects of the amplitude-wavelength ratio, $\alpha$, on the local skin-friction coefficient, the local Nusselt number and the local Sherwood number are shown in Fig. 2(a), (b) and (c) respectively. From Figs. 2(a) and (c), it is seen that, with the increase of $\alpha$, the skin-friction coefficient and the couple stress fluctuate with higher amplitudes. However the maximum values of skin-friction coefficient and the couple stress decrease with increasing values of $\alpha$. On the other hand, we observe from Fig. 2(b) that the amplitude of the local Nusselt number becomes higher for higher $\alpha$. It is because higher value of amplitude-wavelength ratio hinders the flow field that results in decrease to the drag coefficient and the couple stress.
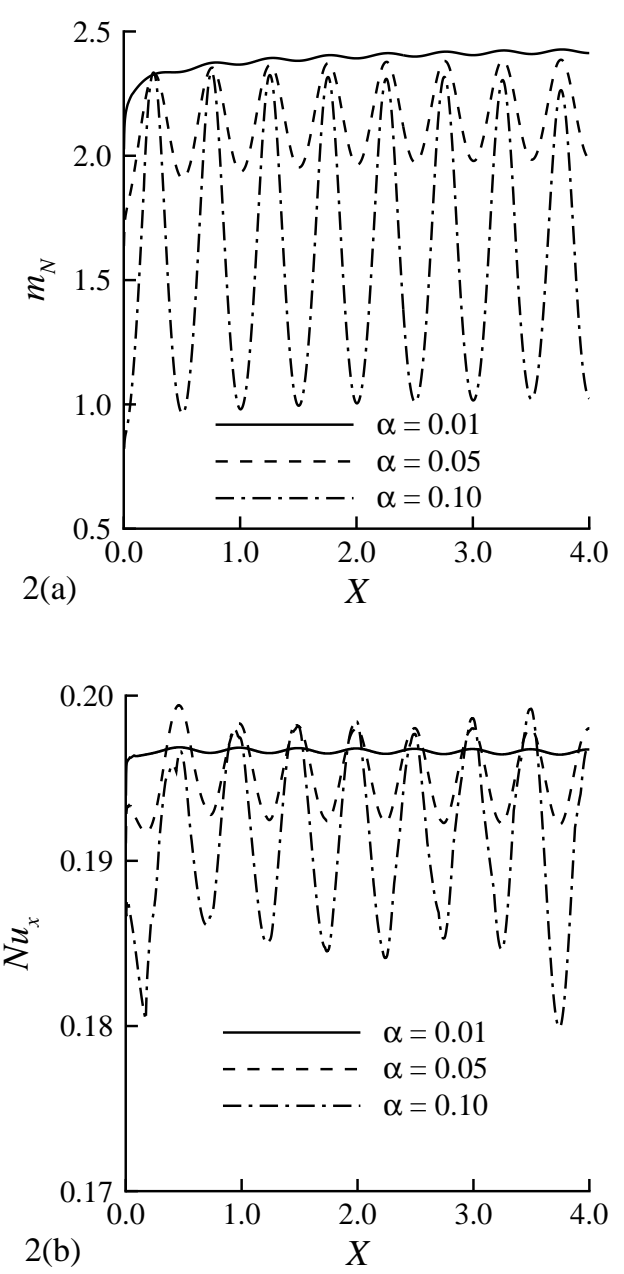


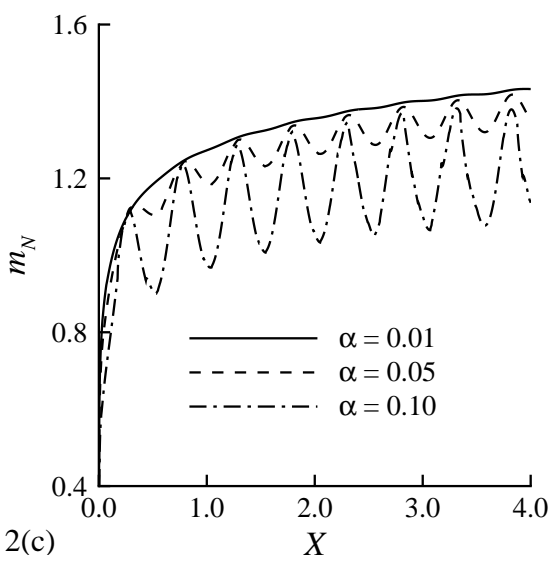

Fig. 2. Effects of the amplitude-wavelength ratio, $\alpha$, on the (a) local skin-friction coefficient, (b) local Nusselt number and (c) local couple stress.

The influences of varying the magnetic parameter, $M$, on the local skin-friction coefficient, the local Nusselt number and the local couple stress are illustrated in Fig. 3(a), (b) and (c) respectively. Results suggest that the local skin-friction, Nusselt number and couple stress decrease with an increase the magnetic parameter, $M$. From the definition of the magnetic parameter, it is found that magnetic parameter increases owing to the increase of the strength of magnetic field. Hence the decrease of the amplitude of the local skinfriction coefficient is the result of increasing the strength of the magnetic field.
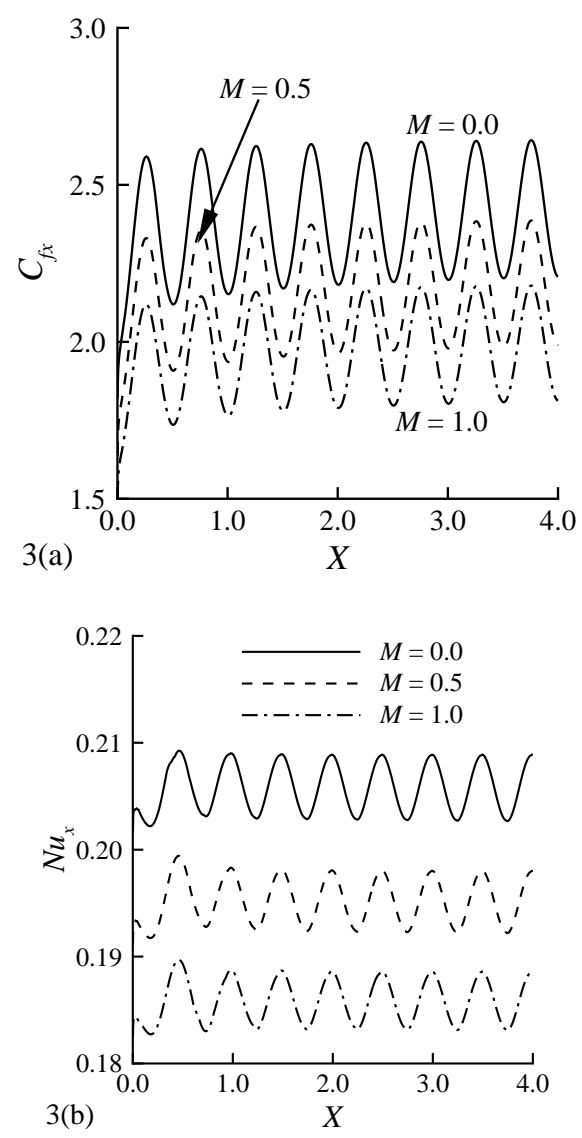

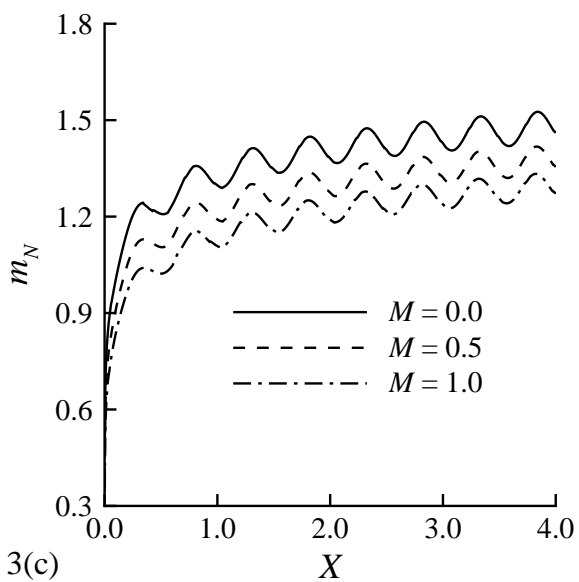

Fig. 3. Effects of the magnetic parameter, $M$, on the (a) local skinfriction coefficient, (b) local Nusselt number and (c) local couple stress.

Figs. 4(a), (b) and (c) depict the effects of the change of the vortex viscosity parameter, $K$, on the local skin-friction coefficient, the local Nusselt number and the local couple stress. We observe from Fig. 4(a) that the local skin-friction coefficient of micropolar fluid $(K \neq 0)$ increases with the increase of the vortex viscosity parameter, $K$, than the Newtonian fluid $(K=0)$. However it is evident from Fig. 4(b) that the local Nusselt number of micropolar fluid $(K \neq$ $0)$ decreases owing to increase of the vortex viscosity parameter, $K$, compared to the Newtonian fluid $(K=0)$.

Fig. 4(c) shows that the couple stress of micropolar fluid $(K$ $\neq 0)$ is greater than the Newtonian fluid $(K=0)$. It can be understood from the definition of the vortex viscosity parameter $K=\kappa / \mu$ which indicates that the value of $K$ becomes higher either the coefficient of viscosity $\mu$ is lower or the coefficient of gyro-viscosity $\kappa$ is higher. On the contrary, it is evident that the skin friction coefficient and the couple stress become stronger with lower $\mu$ and higher $\kappa$, respectively.

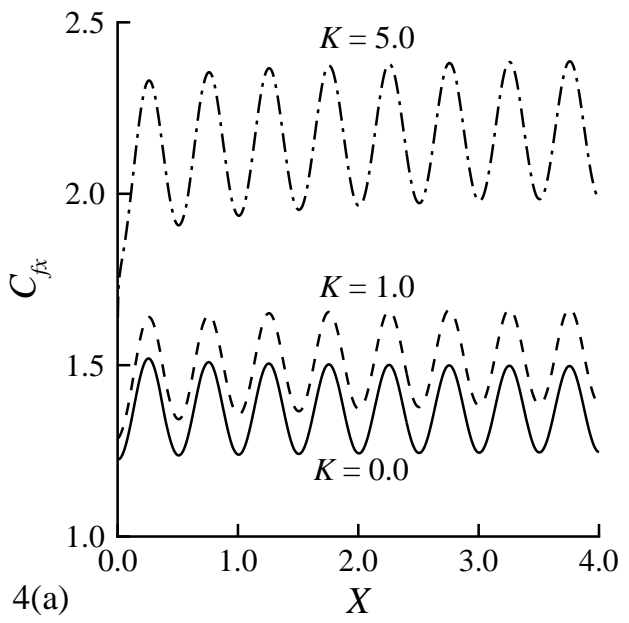



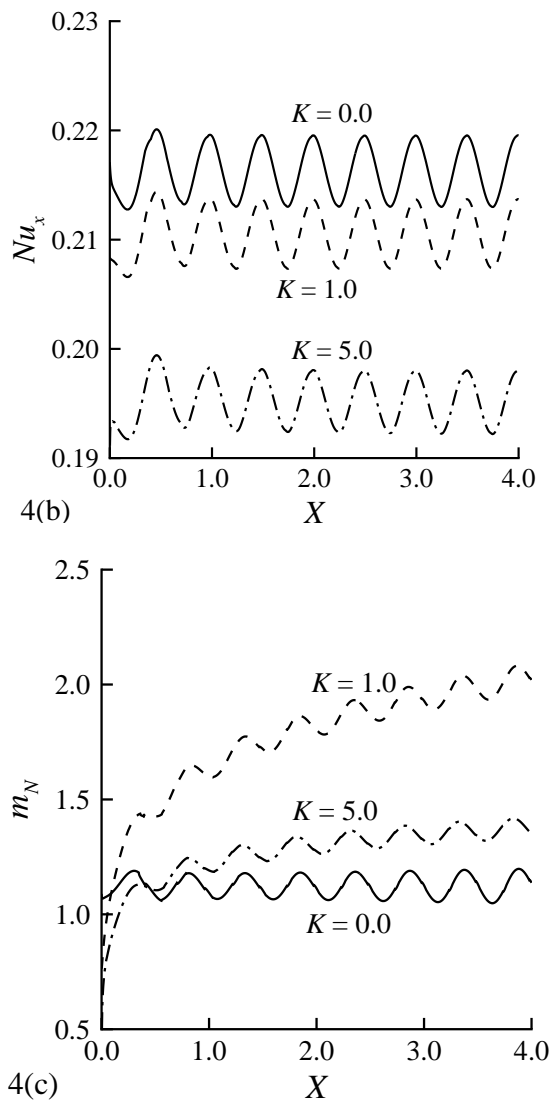

Fig. 4. Effects of the vortex viscosity parameter, $K$, on the (a) local skin-friction coefficient, (b) local Nusselt number and (c) local couple stress.

The effects of the spin-gradient viscosity parameter, $\lambda$, on the local skin-friction coefficient, the local Nusselt number and the local couple stress are demonstrated in Fig. 5(a), (b) and (c) respectively. Results indicate that the local skin-friction coefficient decreases with the increase of $\lambda$. But the Nusselt number and the couple stress are found to increase owing to an increase of $\lambda$. It is also clear from the figures that the amplitudes of oscillation of the local skin-friction coefficient, the local Nusselt number and the local couple stress increase with the values of $\lambda$. This is due to the fact that the couple stress is proportional to $\lambda$ while the drag coefficient decreases for stronger spin-gradient viscosity.

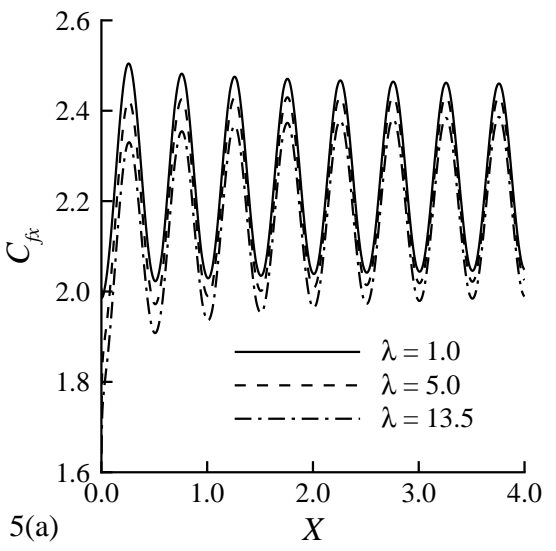

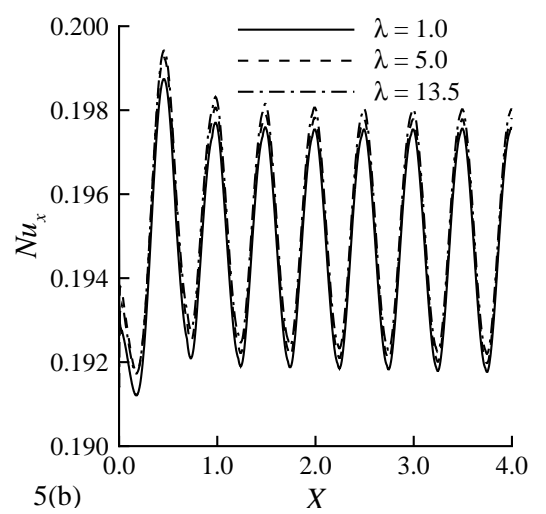

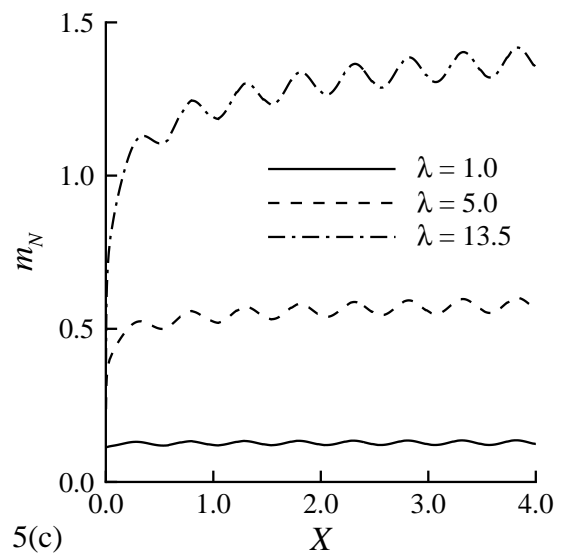

Fig. 5. Effects of the spin-gradient viscosity parameter, $\lambda$, on the (a) local skin-friction coefficient, (b) local Nusselt number and (c) local couple stress.

\section{Conclusions}

The free convection boundary layer flow of an electrically conducting micropolar fluid over a vertical wavy surface has been studied numerically. Results are presented in terms of the local skin-friction coefficient, the local Nusselt number and the local couple stress with the variation of the amplitude wave-length ratio, the magnetic parameter, the vortex viscosity parameter and the spin-gradient viscosity paramter. With the increase of the amplitude wave-length ratio, the local skin-friction coefficient and the couple stress decrease and the Nusselt number increases. Moreover, the Nusselt number and the couple stress increase and the local skin-friction coefficient decreases owing to an increase of the spin-gradient viscosity. The important result is that the magnetic parameter significantly reduces the skin-friction, Nusselt number and couple stress.

\section{References}

1. Eringen, A. C., 1966. Theory of micropolar fluids, J. Math. Mech., 16, 1-18.

2. Eringen, A. C., 1972. Theory of thermomicropolar fluids, J. Math. Appl., 38, 480-495.

3. Ariman, T., M. A. Turk, N. D. Sylvester, 1974. Application of microcontinuum fluid mechanics, Int. J. Eng. Sci., 12, 273-293.

4. Ariman, T., M. A. Turk, N. D. Sylvester, 1974. Micro continuum fluid mechanics review, Int. J. Eng. Sci., 11, 905930. 
5. Yao, L. S., 1983. Natural convection along a vertical wavy surface, ASME J. Heat Transfer 105, 465-468.

6. Chiu, C. P., H. M. Chou, 1993. Free convection in the boundary layer flow of a micropolar fluid along a vertical wavy surface, Acta Mechanica, 101, 161-174.

7. Chiu, C. P., H. M. Chou, 1994. Transient analysis of natural convection along a vertical wavy surface in micropolar fluids, Int. J. Eng. Sci., 32, 19-33.

8. Ishak, A., R. Nazar, I. Pop, 2007. MHD stagnation point flow towards a stretching vertical sheet in a micropolar fluid, Magneto., 43, 83-97.
9. Adhikari, A., 2013. MHD micropolar fluid flow towards a stagnation point on a vertical surface under induced magnetic field with radiative heat flux, Int. J. Engg., Research Tech., 2(8), 135-144.

10. Hossain, M. M., A. C. Mandal, N. C. Roy, and M. A. Hossain, 2013. Fluctuating flow of thermomicropolar fluid past a vertical surface. Applications and Applied Mathematics: An International Journal, 8, 128-150. 